\title{
A New Approach to Develop Collaborative Ontology based BPMN Case Study: Generation of Fiscal Code
}

\author{
Meryam El mrini \\ Ibn Zohr University, IRF-SIC Laboratory, Agadir, 80000, Morocco \\ E-mail: elmrinimeryam@gmail.com \\ El hassan Megder and Mostafa El yassa \\ Ibn Zohr University, IRF-SIC Laboratory, Agadir, 80000, Morocco \\ E-mail: Megderel@yahoo.fr, melyass@gmail.com
}

Received: 02 December 2019; Accepted: 25 December 2019; Published: 08 February 2020

\begin{abstract}
Collaborative platforms are becoming a necessity for enterprises and organizations since they have become extended and have to work with other organizations in joint projects. Knowledge management is considered one of the critical successes of collaborative tools and platforms, especially collaborative ontologies. This paper aims to propose a new approach for developing a collaborative ontology that can be used to support a collaborative platform. Our approach begins with the idea of using a business process model of the collaborative situation, represented in BPMN notation, and to transform it into a collaborative ontology.

Our collaborative process will be modeled collaboratively, and its transformation to an ontology will be done through a first transformation to Subject oriented BPM (S-BPM). We also proposed to validate our collaborative ontology using competency questions that will be formulated at the beginning by domain experts and the ontologist and verified at the end by the ontologist. Fiscal code generation of a newborn was adopted as a case study to prove the importance of this approach.

Compared to other approaches cited in the literature, our approach allows the construction of a collaborative ontology, that does not need enrichment perspectives, and that involves all collaborative partners during its construction process.
\end{abstract}

Index Terms-Collaborative ontology, BPMN, S-BPM, Competency questions.

\section{INTRODUCTION}

According to Enterprise Business Collaboration 2018 Survey Report [1], the use of collaboration tools and platforms highly increased. Main applications on digital platform being of $78 \%$ for knowledge management and $58 \%$ for Office Applications. Influencing performance factors of the digital platform are (Corporate Culture 65,
User adoption 64\%, Budget 38\%).

Knowledge Management (KM) is one of the critical success of collaborative tools and platforms; it improves knowledge sharing and collaborative work, especially between enterprises or organizations due to the new extended environment of enterprises [2].

The development and implementation of a collaborative platform is a difficult problem. Indeed, for these types of projects, interoperability is one of the most challenging requirements to ensure. Amongst many definitions, interoperability is defined as the ability of two or more systems or components to exchange information and to use the information that has been exchanged [ 3].

For supporting collaborative platforms, there is different semantic technologies, in our case we are interested in ontologies, specifically a collaborative ontology. Developing a collaborative ontology that can be used by a collaborative platform has highlighted several challenges and research questions: which techniques can be used for knowledge gathering to populate the ontology? How to take into consideration the business needs of the partners of the collaboration? How to verify the collaborative ontology?

We carried out a literature review of the various works responding to our problem; it allowed us to identify different approaches concerning using ontologies to develop a collaborative platform and collaborative ontology engineering. Several papers in the literature focus on gathering knowledge using frameworks and developing global collaborative ontologies, other works tried to enrich the previous works by adding dynamic concepts to the resulting ontologies, while others proposed a more generic approach allowing a meta modelization of the inter-organizational collaborative process. Indeed, some of them proposed a variety of methodologies, methods, and techniques for developing collaborative ontology. The best work from our perspective is the work that focused on the different methodologies for developing collaborative ontologies. 
In this paper, we have relied on the analysis of the existing literature, and we have noticed several gaps, such as the multiples perspectives of enrichment of the collaborative ontology, the collaborative process modeling was not in a collaborative manner with the intervention of the different actors of the collaboration. The proposed methodologies, for collaborative ontology engineering, do not accurately identify each participant's contribution, and there are no methods to verify the resulting collaborative ontology.

The purpose of the paper is to tackle the above gaps. Our objective is to develop a collaborative ontology applicable to any collaborative situation without the need for enrichment perspectives. We want to base the construction of the collaborative ontology on a business model already existing in the information system of the collaborative partners to be sure to identify the business needs of each partner, to design this model in a collaborative way and use a method to verify the resulting collaborative ontology.

This paper is organized into five sections. The second section deals with the literature review. The third is about the approach that we proposed for developing a collaborative ontology. The fourth section deals with the case studied, the generation of fiscal code, to which our prototype was applied, and the results achieved. The fifth section presents the conclusion and perspectives of this work.

\section{LITERATURE REVIEW}

We can roughly classify the literature related to this paper into three main groups: works that introduced, for the first time, the semantic level, represented by ontologies, to collaborative platforms. Works that proposed to enrich the ontology resulting from the work of the first group, by metamodeling and verifying the business process resulting from the transformation of this ontology and, finally works dealing with collaborative ontology engineering.

Knowledge is a more abstract level of a collaborative information system; they represent the business level. The work of [4] defined a knowledge-based system (Kbs), to produce a valid collaborative business process model to be executed within a collaborative platform. A collaborative ontology-based approach has been developed for dealing with knowledge representation and reasoning, which is the core of the Kbs. The originality of this work is the introduction of the notion of collaborative ontology in the development of collaborative platforms, but the resulting ontology needed some enrichment perspectives because of the lack of some concepts concerning partners. This work forms the basis of our approach, but without the need for enrichment perspectives.

Reference [5] continued the work of [4] by providing agility in operation to be able, not only to design a collaborative information system of mediation but to make evolutionary maintenance of reverse engineering and engineering adapted to the continual evolution of the need. This work did not focus on enriching the resulting collaborative ontology but worked on continuing the previous work by transforming the collaborative ontology into a business process. The relevance of this work towards our objective is the mapping of an ontology to a business process, which is one of our objectives in the other sense of transformation.

Reference [6] enriched the works of [4] by automating the generation of the transformation of the characterization and of a model of collaborative situation in a model of mapping, cartography, of collaborative business processes. This work helped us to think about designing a collaborative business process modeling our collaborative situation and the different collaborative partners and their tasks.

As a supplement to these works, [7] developed the transformation of business processes in feasible technical processes. This passage consists of annotating the selected services semantically, among the available ones, modeled in the various processes, those who cover the features of the activities jobs, however, this work was focused on transforming the business process resulting from the collaborative ontology into executable processes, but our work is more concerned with the development of the collaborative ontology based on a business process the inverse engineering of this work

Reference [8] treated a particular type of collaboration that of the case of crisis by proposing a solution considered as an information system of mediation, within the framework of the project ISyCri, conceived an information system for several partners who have to solve, or at least reduce a crisis situation in which they are involved. A crisis situation is a situation that requires collaboration to solve a problem its solution is a common objective between the different parties involved in the situation.

Reference [9] based itself on the works of [4], while enriching the ontology quoted this work, by adding dynamic concepts in the ontologies, transformation rules of the collaborative process, and a set of services, this work corresponds to one of our objectives that of the enrichment of collaborative ontology, except that our work is based on a business model to enrich our collaborative ontology.

The second category of related works was interested in the collaborative business process resulting of the works of [4], by combining the work of [4] and that of [9], to propose a more generic approach allowing a meta modelization of the inter-organizational collaborative process [10], this work corresponds to our objective to model our business process in a collaborative way between organizations concerned by the collaborative situation understudy. This approach starts of the principle that within an inter-organizational collaboration, the various actors do not still have the same point of view on the notion of business process, each evolving in the environment, its universe of skill and each having to sound his model of a business process [11], from this work we decided that our business process will be modelled with the participation of each collaboration 
partner, as each partner has to model its tasks.

Reference [4] provides a model of the collaborative process, the latter, which can be constrained by shared resources or by processing times, must be checked before being handed over to the collaboration execution platform. In the literature, several techniques have been adopted for verification of the process model, [12, 13] used the technique of model checking for the verification of business processes. [14] explored the structural theory of Petri nets to approach the modeling and verification of business processes by improving a chosen algorithm in the literature. The work of [15] adopts an approach based on automatons as well as formal composition, and [16] uses an approach of the check based on the transformation(processing) of graphs, as the verification methods mentioned above are not reliable in the case of a collaboration, we are going to base the verification of our collaborative business process on the use of a software that we will detail in the section dedicated to the implementation of our approach.

The last category of related works was a focus on collaborative ontology engineering by proposing a great variety of methodologies [17]. Although there is increased flexibility in being able to switch between roles, practice shows that there is, at a task level, is differentiation of roles [18].These methodologies have helped us to structure our methodology for developing our collaborative ontology, but these methodologies cited in the literature encounter a problem of role ambiguity among participants, every participant is an active ontology editor, but whether they created new classes, properties, or instances differed significantly.

Other research emphasis on Semantic Wikis, such as Semantic MediaWiki [19]. In spite of this, this software is open to everybody and has a "human-readable interface to ontologies" [20]; the knowledge base is limited to a specific domain, which is not working for our case that of a collaborative situation applicable to different domains. The work of [21] analyzed editing actions in various Semantic MediaWiki communities, based on their results, building structured data based on Wiki-software, but it is a very individual behavior and, in our case, we need a collaborative work with different partners and each one has its specific tasks.

Freebase is probably the most prominent example of an open system in which contributors can build a structured dataset to describe topics of interest and create collections of connected topics [22]. However, existing patterns of participation and how people dealt with ontological primitives will remain unknown.

Although many efforts have been carried on so far in order to develop a collaborative ontology, we noticed several gaps; the first works tried to populate ontology using user frameworks, which generated a lack of concepts and, consequently, perspectives of ontology enrichment. The second category of work focused on the collaborative process, resulting from collaborative ontology, more than on the development of the collaborative ontology, so there is no verification of business needs that they are well defined and taken into consideration. The last category of work concerned the different methodologies for the development of the collaborative ontology; these methodologies encounter several difficulties: It is not clear at what level the participation of each collaborator is and that they have the tasks with which they have contributed to the collaboration. The Semantics wiki is open to everyone, while in a collaborative situation, the collaborators are well designated, at the wiki levels there is individual rather than collaborative work. There is no verification of the collaborative ontology if it meets the business needs of collaboration defined at the beginning.

\section{THE PROPOSED APPROACH}

The main goal of our approach is to provide an improved and optimized solution to develop a collaborative ontology that can be used by a collaborative platform. Ontologies are a means of capturing and representing knowledge, [23] introduces the notion of ontology as "an explicit specification of a conceptualization." This definition has been a little bit modified by [24].

Our idea started with the transformation of a business process to a collaborative ontology. Several definitions of business process have been proposed in literature, a business process is a structured, measured set of activities designed to produce a specific output for a particular customer or market, hence a process is a specific work activity, in order, beyond time and space, clearly defined inputs and outputs with a beginning and an end [25].we opted for the use of a business process to populate our collaborative ontology for two reasons: business needs are already well identified and defined, participants are well designated along with their tasks and the interactions between the participants.

Fig. 1 represents the prototype of our approach, it explains the different phases for the transformation of a business process, represented in the BPMN, into a collaborative ontology. Our prototype starts with the modeling of the business process collaboratively by domain experts and the BPM consultant, which belongs to different organizations, this field changes according to the situation of collaboration. The role of the BPM experts is to validate the modeled BPMN process and verify its compliance with the BPMN meta-model. Each domain expert models its tasks, in that way, we ensure that each participant contributes to the modeling and can modify the process. The modeling of this business process is done using a software that we will detail during the implementation phase. to verify the collaborative ontology, resulting from the approach, we have chosen to use the method of Competency questions, these questions must be defined by domain experts and the ontologists at the beginning before the ontology is generated, these questions are formulated following specific patterns. The transformation from BPMN to owl will be developed through Subject oriented BPM(S-BPM) that we will explain in details in the following section. The resulting 
collaborative ontology will be verified using Competency Questions already formulated.

The prototype of approach is divided into four steps: the modeling of the collaborative business process in a collaborative manner, the formulating, by the domain expert, of the competency question that our collaborative ontology should answer, the transformation from BPMN to OWL, and the verification of the ontology using competency questions.

Our approach started with a BPMN process modeled with the intervention of the domain expert and bpm consultant using Bonita studio [26], the competency questions should be formulated by the domain expert and the ontologist using different patterns. The BPMN process will be transformed into an ontology through a first transformation to Subject Oriented BPM (S-BPM) using a Java Program [27]. The last step is the verification of the collaborative ontology by the ontologist using Competency questions defined at the beginning by the domain expert.

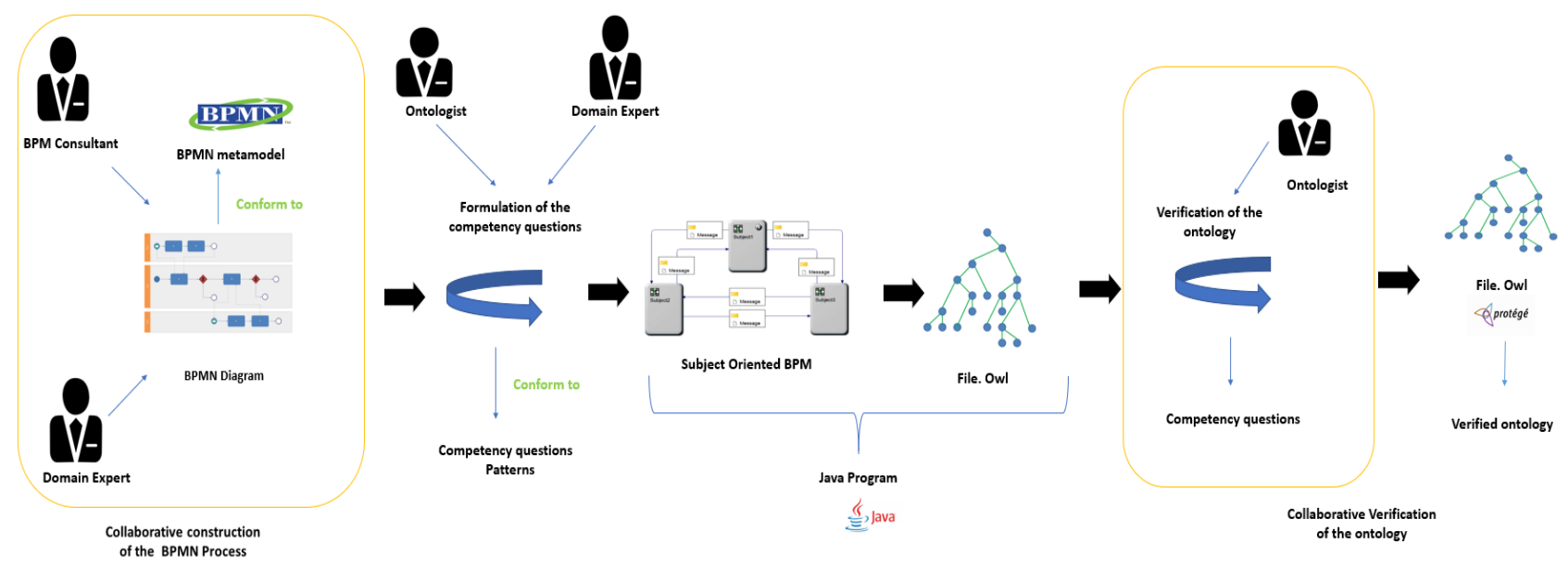

Fig.1. Prototype of the proposed approach

\section{Collaborative Ontology: Generation of Fiscal CODE OF A NEWBORN AS CASE STUdY}

\section{A. Study Case}

Our case study comes from a study related to public administration in Italy, describing the procedure for managing the attribution of the Italian tax code for newborns, which aims at registering citizens' birth data. The data must be stored both in the municipality and in the national register (SAIA) since the newborns are Italian citizens living in a given municipality. This process requires the collaboration of several actors, as well as the generation of some crucial data as in our case its fiscal code.

This case is an adequate case study for our work focusing on the collaboration between heterogeneous Organizations. This case highlights two challenges, combining three different information systems (hospitals, municipality and SAIA system) and a challenge of semantic heterogeneity due to the use of knowledge coming from different institutes and public domains. We will apply our prototype on this case to create a collaborative ontology to solve the problem of semantic heterogeneity and heterogeneity of information systems using different technical structures.

\section{B. The Business Process}

Our approach was applied to the fiscal code generation process for a newborn in an Italian hospital, considering that in Italy every person has a fiscal code. The process model, of this case, represented in the BPMN notation is represented in the work of [28] and illustrated in Fig.2.

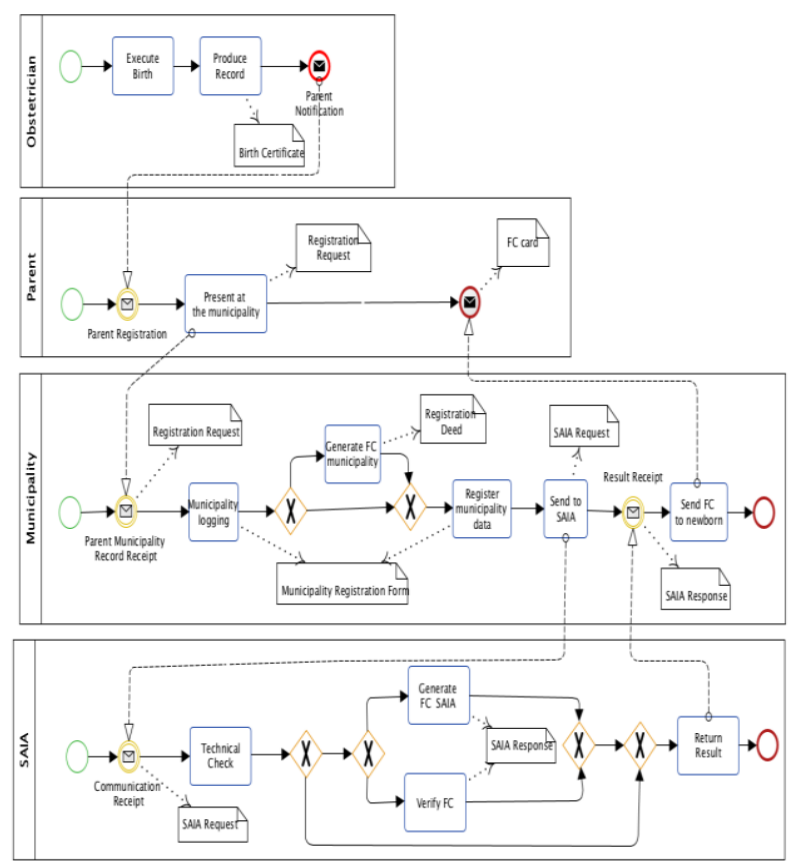

Fig.2. The generation process of fiscal code represented in the BPMN notation [28]

The generation of the fiscal code process requires collaboration between different partners: the hospital, the 
parents, the municipality, and the national information system (SAIA). This case is technically relevant, because these partners represent heterogeneous information systems that must collaborate and exchange messages to generate a fiscal code assigned for a newborn.

The process starts with the obstetrician representing the hospital; he will produce a birth certificate and give it to the parents, who will present it to the municipality and request registration. The request will be registered by the municipality, who will generate a tentative fiscal code then send the request to SAIA. SAIA checks the request and generates or validates the fiscal code before returning it to the municipality, which then generates the card for the newborn and sends it to the parents.

\section{The Collaborative Modeling of the BPMN Process}

Fig.3 shows the first step of our approach that is modeling our BPMN process under the open-source tool Bonita Studio, and our participants of collaboration. Bonita Studio offers a module that allows management and collaboration between the different participants in the process modeling, Fig. 4 represents the module Portal that shows the current participant, his tasks performed and those to be done, and he can visualize the whole process.

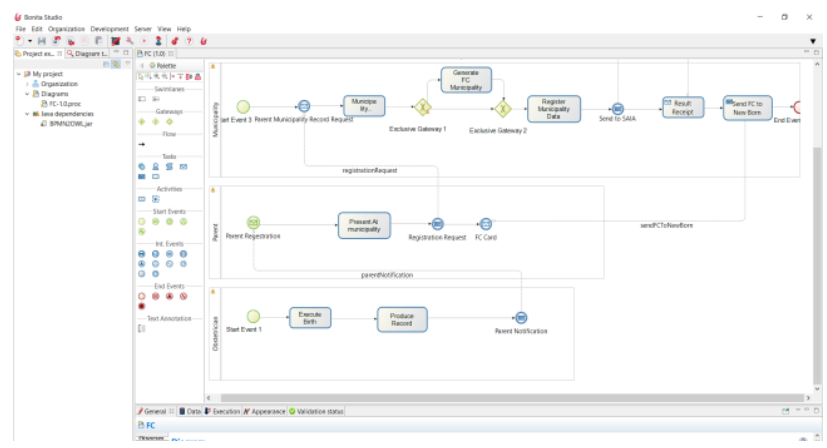

Fig.3. The generation process of fiscal code modeling in Bonita Studio

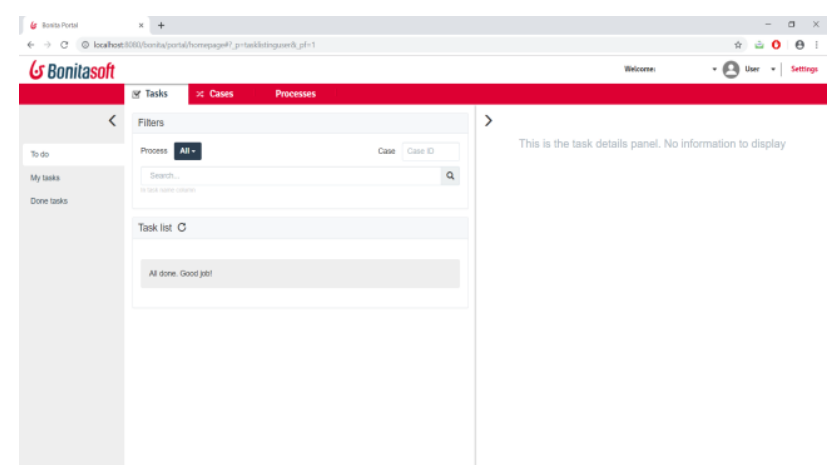

Fig.4. Business process modeling in Bonita Studio Portal

\section{Formulation of the Competency Questions}

Several ontology development methodologies [29, 30, 31] propose the use of Competency Questions (CQs) as ontology requirements. However, traditional methodologies did not describe how to use Competency Questions (CQs). The work of [32] came up with the first approach on how ontology engineers should describe a CQ using axioms and text.
CQs represent user demands for knowledge regarding a domain of discourse. They usually enable developers to define the ontology. Since we are dealing with controlled natural language, we are dealing with some patterns of CQs that we have found, below some of some of them and the questions, concerning our case, that was built following these patterns:

Pattern: From which $+<$ property $>+<$ class $>$ ?

CQ: From which participant Parent receive a Message?

Output: list of classes

Pattern: Is $+<$ individual $>+\mathrm{a}+<$ class $>$ ?

CQ: is Municipality a participant?

Output: true or false.

\section{E. Transformation from BPMN to $O W L$}

The next step of our approach is the transformation from BPMN to OWL. It is divided into two stages, a transformation from BPMN to S-BPM and transformation from S-BPM to OWL. Subject-oriented BPM (S-BPM) is a BPM approach that focuses on the acting elements in a business process, i.e., the subjects. In many natural languages, sentences start with the subject, just as we start with the subject in S-BPM [33], it is a business process modeling language based on the actor system, which has been defined via an OWL ontology.

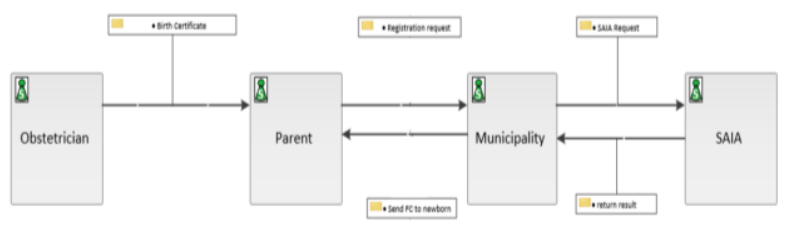

Fig.5. Subject interaction diagram (SID) of S-BPM

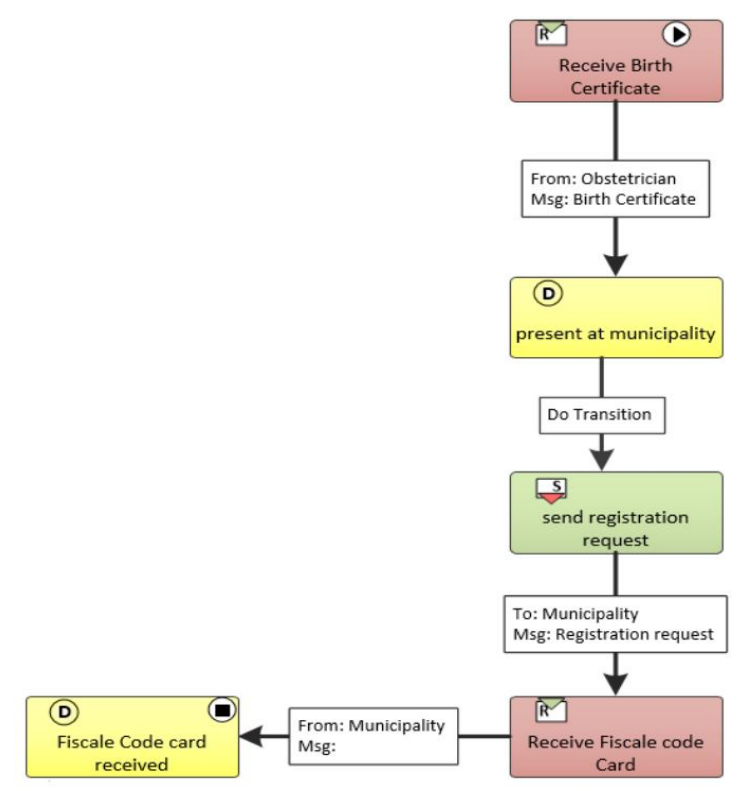

Fig.6. Subject Behavior Diagram (SBD) of S-BPM

Commonly, S-BPM models consist of two types of representations: A Subject Interaction Diagram (SID) and a set of Subject Behavior Diagrams (SBD). Respectively Fig.5. Illustrate the SID of our case, includes subjects and 
messages exchanged between them (Obstetrician, Family, municipality and the SAIA system). Fig.6. represent the SBD of our case that includes the behavior of the subject Municipality, representing the messages exchanged with the other subjects.

Bonita Studio allowed us to integrate our jar file, which provides the transformation From BPMN to OWL. After the transformation, we used Protégé tool to visualize the ontology. Fig.7. Shows the result of the transformation of our BPMN model into an ontology, the main class, subclasses, and some individuals. The classes represent the different tasks represented in the BPMN process and the partners; for example, the individuals of the class participant are the actors of the collaboration (e.g., Municipality).

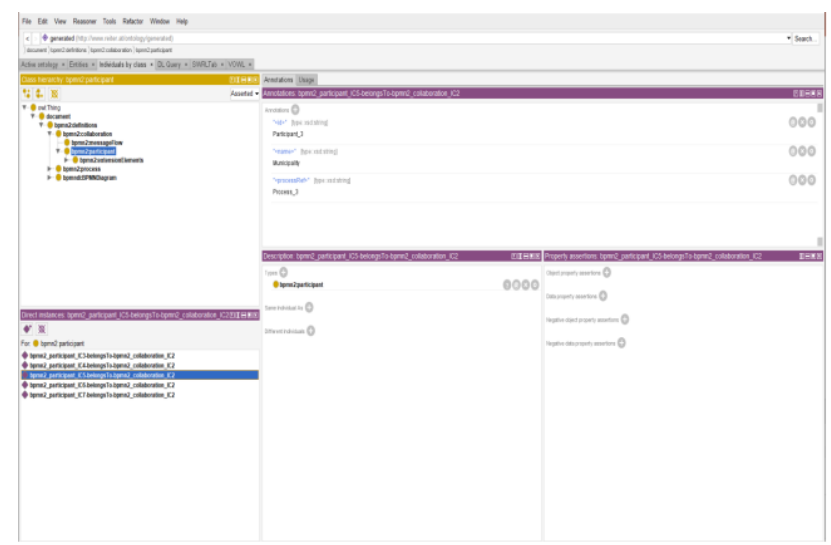

Fig.7. The resulting ontology Main Class, Subclass, and Individual

Fig.8 Shows how this ontology is visualized using VOWL under Protégé. It represents the Main class, subclasses, and the relationships (Object Properties) between these classes. The main classes represent the components of the BPMN process, the participants. The relationships represent the links between the tasks and the messages exchanges between the participants.

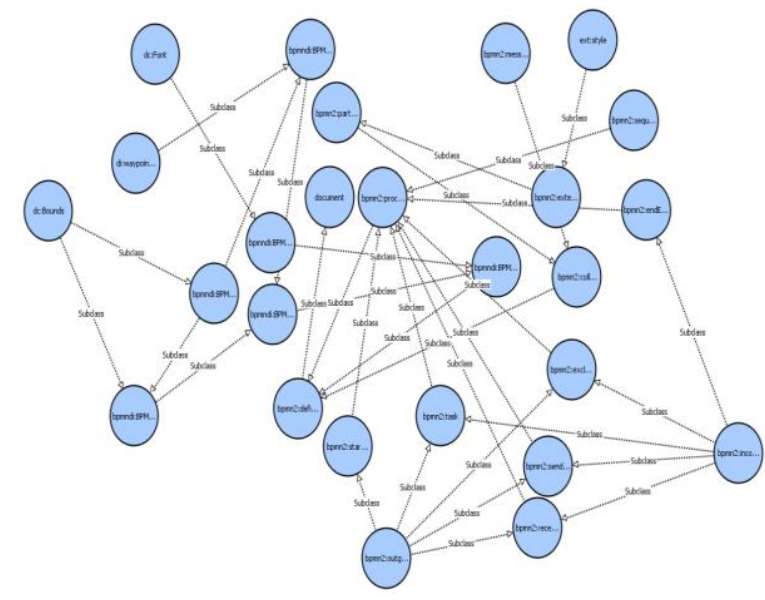

Fig.8. The resulting ontology Visualization using VOWL

\section{F. Verification Of The Ontology}

Table 1 is an evaluation table that shows the answers we have already formulated and the correct answers and those verified after the application of our approach.

Table 1. Results of Verification of the Competency questions

\begin{tabular}{|c|c|c|c|}
\hline $\begin{array}{c}\text { Competency } \\
\text { Question }\end{array}$ & Correct Answer & Verified answer & Correct? \\
\hline $\begin{array}{c}\text { From which } \\
\text { participant } \\
\begin{array}{c}\text { Parent receive } \\
\text { a Message? }\end{array}\end{array}$ & Municipality & Municipality & YES \\
\hline $\begin{array}{c}\text { Is Municipality } \\
\text { a participant? }\end{array}$ & True & True & YES \\
\hline
\end{tabular}

The results shown in the previous table show that our resulting ontology does not need to be enriched by concepts, and it has responded to the competency questions already formulated at the beginning of the collaborative ontology development process by ontologists and the domain experts.

\section{G. Discussion}

Our first objective was to propose a new collaborative ontology development method and apply it to the case of the fiscal code generation of a newborn in an Italian hospital, considering that in Italy every person has a fiscal code. The study found that we will need collaboration between different organizations including the hospital, the municipality and the SAIA system, which is a challenge since these organizations represent heterogeneous information systems at the technical and semantic levels.

To develop our collaborative ontology we used a business process represented in BPMN, which ensured that each organization in our collaborative situation could model its tasks and visualize the entire collaborative process, via the Bonita studio tool, which is an advantage, over the works cited in the literature review, that relied on Frameworks to collect knowledge from participants and populate the ontology, resulting in a lack of concepts in the collaborative ontology.

Our approach ensured that we could know for each task its owner, which solved the problem of MediaWiki being open to everyone or constituting individual behavior. The resulting ontology contains concepts representing the different actors in the collaboration as well as the different messages exchanged between the actors represented by the relationships between the concepts. We used the method of competency questions to check our ontology. The resulting collaborative ontology was able to answer the competency questions that we formulated at the beginning of our development process, which shows that our ontology will not need to be enriched for better collaboration. Our approach has limitations, as it does not support other sources of knowledge to populate the collaborative ontology outside of the business processes represented in BPMN and also the automation of ontology verification.

\section{CONCLUSION AND PERSPECTIVES}

Collaborative platforms and ontologies become more important and related, to support a collaborative platform 
using ontologies, this research paper aimed to propose a new approach for developing a collaborative ontology.

Our approach enables the participant of a collaborative situation to model a collaborative business process collaboratively represented in BPMN, which allows us to identify the business needs of each collaboration partner and therefore avoid a lack of concepts in the resulting ontology that can lead to unsatisfactory collaboration, and to transform this business process into a collaborative ontology through a first transformation to S-BPM.

The resulting ontology was verified using competency questions that were formulated at the beginning by the domain expert. The generation of the fiscal code of a newborn used as a case study, since it requires the collaboration between four participants, after modeling the business process of this case using Bonita Studio, the BPMN process were transformed into a collaborative ontology that we visualized using Protégé and VOWL, and we verified using the competency questions.

Our approach has made it possible to develop a collaborative ontology based on a business process; it represents the business needs of each participant, knowing precisely how each participant contributes to collaborative ontology. The participants can modify the collaborative business process according to their needs and tasks, which will result in the change of the collaborative ontology. The collaborative ontology is verified using competency questions formulated by domain experts from the beginning and verified at the end of the collaborative ontology development process.

Our approach can be used for any collaborative situation between heterogeneous collaborative information systems, based on ontologies and we will quote in the following paragraph the future perspectives of our work.

The case study of fiscal code generation is a good example of a situation in need of collaboration modeled as a process between heterogeneous partners with specific tasks for each partner. This case allowed us to apply our prototype and to have relevant results that verify our approach to developing collaborative ontologies based on a business process represented in BPMN.

We have prospects of enriching our approach by using other models to populate our ontology, such as the UML class diagram and alignment with other ontologies of domains to enrich our ontology better collaborative.

\section{ACKNOWLEDGMENT}

The authors wish to thank the Laboratory of applied ontology, especially professor Stefano Borgo, in Trento Italy, for hosting our corresponding author as a research visitor for last July and their contribution to the development of our prototype. And we would like to show our gratitude to the National Centre for Scientific and Technical Research (Morocco) for offering an excellence research grants to our corresponding author so she can undertake her research.

\section{REFERENCES}

[1] https://www.enterprise-communication-hub.com/wpcontent/uploads/2017/10/Enterprise_Business_Collaborati on_Survey_report_2018-1.pdf (Accessed: 09 November 2019).

[2] R. Dieng-Kuntz and N. Matta. Knowledge Management and Organizational Memories. Kluwer Academic Publishers, 2002. DOI:10.1007/978-1-4615-0947-9.

[3] IEEE Standard Computer Dictionary: A Compilation of IEEE Standard Computer Glossaries," in IEEE Std 610, vol., no., pp.1-217, 18 Jan. 1991 DOI: 10.1109/IEEESTD.1991.106963.

[4] R. Vatcharaphun, "Knowledge-based system for collaborative process specification", $\mathrm{PhD}$ Thesis, Toulouse University, Toulouse, France, 2009.

[5] S. Truptil, "Study of the approach to interoperability by mediation within the framework of a dynamic of collaboration applied to crisis management", PhD Thesis, INPT - EMAC, France, 2011.

[6] M. Wenxin, "Business and logical characterization of a collaborative situation", $\mathrm{PhD}$ Thesis, School of Mines in Albi, France, 2012.

[7] N. Boissel-Dallier, "Help in designing a Collaborative Mediation Information System: from business process mapping to executable", $\mathrm{PhD}$ Thesis, Institut National Polytechnique of Toulouse, France, 2012.

[8] S. Truptil, F. Bénaben, N. Salatge, C. Hanachi, V. Chapurlat, J. Pignon, and Pingaud, "Mediation Information System Engineering for Interoperability Support in Crisis Management", Enterprise Interoperability IV, pp. 187-197, 2010.

[9] S. SAIB, R. BENMOUSSA, and K. BENGOUD, "Modeling of mediation system for enterprise systems collaboration through MDA and SOA", PhD Thesis, Information logistic and production systems Team, National School of applied sciences (ENSA), Cadi Ayyad University-Marrakech, 2013.

[10] K. Semar-Bitah and K. Boukhalfa, "Towards an Interorganizational Collaboration Network Characterization", Modelling and implementation of Complex Systems, pp. 223-248, 2016.

[11] A. Abbassene and Z. Alimazighi, "Towards a Cloud Platform for Inter-Organizational Workflows Integration", Dayes of students ESI (JEESI'14), Alger, 2014.

[12] Z. Sbai, A. Missaoui, K. Barkaoui, and R.B. Ayed, "On the verification of business processes by model checking techniques, Proceeding of the second International Conference on Software Technology and Engineering", October 3-5, 2010.

[13] Z. Sbai, K. Barkaoui, "Formal Verification Collaborative Workflow Processes", Proceedings of Francophone Conference on Collaborative Systems", Sousse, Tunisia, 2012.

[14] Z. Sbai, "Contribution to Workflow Modeling and Process Verification", PhD Thesis, CNAM (National Conservatory of Arts and Crafts), France, 2010.

[15] L. E. Morales, "Business process verification using a Formal Compositional Approach and Timed Automata", Proceedings of XXXI Latin American Computing Conference (CLEI), Venezuela, October 7-11, 2013.

[16] R. ElMansouri, "Modelling and Verification of business processes in virtual enterprises" $\mathrm{PhD}$ Thesis, University Mentouri Constantine, Alger, 2009.

[17] E. Simperl and M. Luczak-Roesch," Collaborative Ontology Engineering: A Survey", Knowledge Engineering Review, 
2012.DOI :10.1017/S0269888913000192.

[18] S. Falconer, T. Tudorache and N. Noy, "An analysis of collaborative patterns in large-scale ontology development projects", Proceedings of the Sixth International Conference on Knowledge Capture,2011, p 25-32. DOI:10.1145/1999676.1999682.

[19] M. Krötzsch et al. Semantic Wikipedia. Journal of Web Semantics, 5(4):251-261, 2007.

[20] F. Bry, S. Schaffert, D. Vrandevci'c and K. Weiand, "Semantic Wikis: Approaches, Applications, and Perspectives. In: Eiter T., Krennwallner T. (eds) Reasoning Web", Semantic Technologies for Advanced Query Answering, Reasoning Web 2012. Lecture Notes in Computer Science, vol 7487. Springer, Berlin, Heidelberg, 2012.DOI: https://doi.org/10.1007/978-3-642-33158-9_9.

[21] K. Bollacker, R. Cook, P. Tufts," Freebase: a shared database of structured general human knowledge", Proceedings of the 22nd AAAI Conference on Artificial Intelligence. 2007, 1962-1963

[22] K. D. Bollacker, C. Evans, P. Paritosh, T. Sturge, J. Taylor,"Freebase: a collaboratively created graph database for structuring human knowledge", SIGMOD Conference 2008: 1247-1250.

[23] Studer (R.), Benjamins (V.R.), Fensel (D.): Knowledge Engineering: Principles and Methods. Data \& Knowledge Engineering. 25: 161-197(1998).

[24] S. Truptil, "Study of the approach to interoperability by mediation within the framework of a dynamic of collaboration applied to crisis management", $\mathrm{PhD}$ Thesis in in industrial systems, INPT - EMAC, France ,2011.

[25] T.H. Davenport, "Process innovation: reengineering work through information technology", Harvard Business Press, 1993.

[26] https://fr.bonitasoft.com/(Accessed 25 October 2019).

[27] https://github.com/Robert62/BPMN_S-BPMOntology(Accessed 29 October 2019)

[28] G. Adamo, S. Borgo, C. Di Francescomarino, C. Ghidini and N. Guarino, "On the Notion of Goal in Business Process Models", (eds) AI*IA 2018 - Advances in Artificial Intelligence. AI*IA 2018. Lecture Notes in Computer Science, vol 11298. Springer, Cham,2018. DOI: 10.1007/978-3-030-03840-3_11

[29] M. Uschold, and M. Gruninger, M, "Ontologies: principles, methods and applications", The Knowledge Engineering Review, 11:93-136,1996.

[30] M. Fernández-López, A. Gómez-Pérez and N. Juristo," METHONTOLOGY: From Ontological Art Towards Ontological Engineering”. AAAI-97 Spring Symposium Series, 24-26 March 1997, Stanford University, EEUU.

[31] M.C. Suárez-Figueroa, A. Gómez-Pérez and M. Fernández-Lópe, "The NeOn Methodology for Ontology Engineering", Ontology Engineering in a Networked World. Springer, Berlin, Heidelberg, 2012.DOI: 10.1007/978-3-642-24794-1_2

[32] M. Fox and M. Grüninger," Ontologies for Enterprise Integration", 1994, p 82-89.

[33] A. Fleischmann, "What Is S-BPM? “,2010, 85106.DOI :10.1007/978-3-642-15915-2_7.

\section{Authors' Profiles}

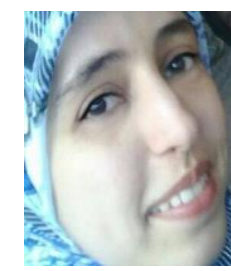

Meryam El mrini is a $\mathrm{PhD}$ student at Ibn Zohr University, Faculty of science, she obtained she obtained a master degree in information system engineering Faculty of Science Semlalia-Marrakech, Morocco in 2016. Her research interests focus on knowledge management, information system modelling and business process

management.

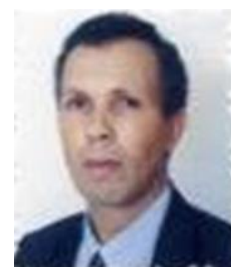

El Hassan Megder is a teacher-researcher at the National School of Commerce and Management. He holds a DES and a national Doctorat in Computer Science. He has been a teacher for 39 years. He has taken on several teaching responsibilities. Several papers and articles have since been published. Its research focus touches on several disciplines of computer science and Artificial Intelligence. Several topics have been addressed, whether in publications or in the framework of multidisciplinary projects, namely: information systems and security, knowledge engineering, the representation of knowledge using ontologies and e-learning.

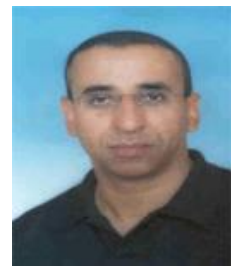

Mostafa EL YASSA is a Professor of informatics at the Faculty of Sciences, University Ibn Zohr, Agadir, Morocco. He received a Doctorat in Informatics and Statistics in 1984 from Nancy University (France) and a PhD degree in Pattern recognition by pretopologique approach from Faculty of sciences, University Ibn Zohr, in 2007. His research areas include: Pattern recognition, pretopologique approach, verification of Handwritten Signatures, image analysis and document processing.

How to cite this paper: Meryam El mrini, El hassan Megder, Mostafa El yassa, "A New Approach to Develop Collaborative Ontology based BPMN Case Study: Generation of Fiscal Code", International Journal of Information Technology and Computer Science(IJITCS), Vol.12, No.1, pp.1-8, 2020. DOI: 10.5815/ijitcs.2020.01.01 